\title{
Persistent residential burning-related primary organic particles during wintertime hazes in North China: insights into their aging and optical changes
}

Lei Liu et al.

Correspondence to: Weijun Li (liweijun@zju.edu.cn)

The copyright of individual parts of the supplement might differ from the CC BY 4.0 License. 
Table S1. Detailed information on individual particle samples collected at the Gucheng rural and Beijing urban sites.

\begin{tabular}{|c|c|c|c|c|c|c|c|c|}
\hline Location & Date & $\begin{array}{c}\text { Local } \\
\text { time }\end{array}$ & $\begin{array}{c}\text { Sampling } \\
\text { duration (s) }\end{array}$ & $\begin{array}{c}\text { Temp. } \\
\left({ }^{\circ} \mathrm{C}\right)\end{array}$ & $\begin{array}{l}\mathrm{RH} \\
(\%)\end{array}$ & $\begin{array}{c}\text { Pressure } \\
(\mathrm{hPa})\end{array}$ & $\begin{array}{c}\mathrm{WS} \\
\left(\mathrm{m} \mathrm{s}^{-1}\right)\end{array}$ & Pollution level \\
\hline Gucheng & 21 Nov. 2016 & $12: 00$ & 180 & -0.6 & 100 & 1018 & 4 & Clean \\
\hline \multirow[t]{11}{*}{ rural site } & 22 Nov. 2016 & 8:06 & 180 & -4.7 & 97 & 1012 & 0.9 & Clean \\
\hline & 22 Nov. 2016 & $19: 22$ & 120 & -3.8 & 47 & 1036 & 0.6 & Heavy (Early stage) \\
\hline & 23 Nov. 2016 & $10: 50$ & 15 & 0.9 & 51 & 1034 & 0.6 & Heavy (Early stage) \\
\hline & 24 Nov. 2016 & 8:06 & 15 & -6.4 & 94 & 1028 & 0 & Heavy (Middle stage) \\
\hline & 25 Nov. 2016 & $3: 00$ & 15 & -2.0 & 79 & 1022 & 1.6 & Heavy (Middle stage) \\
\hline & 25 Nov. 2016 & $8: 07$ & 10 & -4.7 & 93 & 1023 & 0 & Heavy (Middle stage) \\
\hline & 25 Nov. 2016 & $15: 19$ & 10 & 1.4 & 77 & 1022 & 1.6 & Heavy (Late stage) \\
\hline & 25 Nov. 2016 & $20: 44$ & 10 & 1.1 & 84 & 1022 & 1.7 & Heavy (Late stage) \\
\hline & 26 Nov. 2016 & $3: 00$ & 8 & -1.4 & 95 & 1018 & 0 & Heavy (Late stage) \\
\hline & 26 Nov. 2016 & $8: 15$ & 8 & -1.9 & 98 & 1018 & 0 & Heavy (Late stage) \\
\hline & 26 Nov. 2016 & $12: 27$ & 10 & 5.5 & 70 & 1017 & 0.8 & Heavy (Late stage) \\
\hline Beijing & 22 Nov. 2016 & 19:00 & 60 & -1.8 & 33 & 1039 & 0.5 & Clean \\
\hline \multirow[t]{6}{*}{ urban site } & 24 Nov. 2016 & 19:00 & 15 & 2.9 & 41 & 1026 & 0.9 & Moderate \\
\hline & 25 Nov. 2016 & $12: 00$ & 20 & 3.8 & 42 & 1026 & 1.5 & Moderate \\
\hline & 25 Nov. 2016 & $22: 00$ & 10 & 3.1 & 72 & 1024 & 0 & Heavy \\
\hline & 26 Nov. 2016 & 9:00 & 10 & 2.4 & 75 & 1022 & 0 & Heavy \\
\hline & 26 Nov. 2016 & $16: 00$ & 10 & 7 & 45 & 1019 & 0 & Heavy \\
\hline & 26 Nov. 2016 & $22: 00$ & 10 & 3.8 & 71 & 1020 & 0 & Heavy \\
\hline
\end{tabular}


Table S2. Summary of the average meteorological parameters and mass concentrations of $\mathrm{PM}_{2.5}$, aerosol chemical species, organic aerosol (OA) factors, and gaseous pollutants in different periods at the Gucheng rural and Beijing urban sites.

\begin{tabular}{|c|c|c|c|c|c|c|c|}
\hline & \multicolumn{4}{|c|}{ Gucheng rural site } & \multicolumn{3}{|c|}{ Beijing urban site } \\
\hline & \multirow{2}{*}{ Clean } & \multicolumn{3}{|c|}{ Heavily polluted } & \multirow{2}{*}{ Clean } & \multirow{2}{*}{$\begin{array}{l}\text { Moderately } \\
\text { polluted }\end{array}$} & \multirow{2}{*}{$\begin{array}{l}\text { Heavily } \\
\text { polluted }\end{array}$} \\
\hline & & Early stage & Middle stage & Late stage & & & \\
\hline \multicolumn{8}{|c|}{ Meteorological parameters } \\
\hline$T\left({ }^{\circ} \mathrm{C}\right)$ & -1.5 & -2.9 & -1.6 & 0.53 & -2.0 & 2.1 & 3.9 \\
\hline RH $(\%)$ & 97 & 63 & 65 & 78 & 58 & 48 & 64 \\
\hline WS $\left(\mathrm{m} \mathrm{s}^{-1}\right)$ & 2.8 & 0.51 & 0.53 & 0.50 & 1.4 & 0.38 & 0.28 \\
\hline$P(\mathrm{hPa})$ & 1013.5 & 1032.6 & 1026.9 & 1019.6 & 1029.1 & 1026.5 & 1021.5 \\
\hline \multicolumn{8}{|c|}{$P_{2.5}$ and chemical components ${ }^{a}\left(\mu \mathrm{g} \mathrm{m}^{-3}\right)$} \\
\hline $\mathrm{PM}_{2.5}$ & 39.8 & 288.3 & 312.3 & 396.8 & 10.8 & 111.0 & 281.0 \\
\hline $\mathrm{OM}$ & 19.8 & 185.1 & 175.4 & 217.2 & 5.7 & 44.4 & 82.8 \\
\hline $\mathrm{SO}_{4}^{2-}$ & 2.0 & 13.6 & 17.3 & 41.2 & 2.0 & 13.9 & 51.3 \\
\hline $\mathrm{NO}_{3}^{-}$ & 2.4 & 11.3 & 14.7 & 30.8 & 1.9 & 19.7 & 41.0 \\
\hline $\mathrm{NH}_{4}^{+}$ & 1.4 & 11.5 & 13.9 & 28.2 & 1.1 & 9.8 & 24.2 \\
\hline $\mathrm{Cl}^{-}$ & 1.2 & 11.1 & 9.8 & 15.9 & 0.46 & 7.2 & 14.7 \\
\hline EC & 1.4 & 8.9 & 8.7 & 10.9 & l & 1 & / \\
\hline $\mathrm{K}^{+}$ & 0.36 & 1.6 & 1.5 & 2.7 & / & l & / \\
\hline $\mathrm{Na}^{+}$ & 0.63 & 0.41 & 0.42 & 0.92 & / & / & / \\
\hline $\mathrm{Mg}^{2+}$ & 0.08 & 0.18 & 0.15 & 0.28 & / & / & / \\
\hline $\mathrm{Ca}^{2+}$ & 0.75 & 1.1 & 0.84 & 1.8 & / & I & / \\
\hline \multicolumn{8}{|c|}{ OA factors ${ }^{b}\left(\mu \mathrm{g} \mathrm{m}^{-3}\right)$} \\
\hline FFOA & I & I & I & I & 1.0 & 9.2 & 15.6 \\
\hline $\mathrm{COA}$ & l & / & / & / & 1.1 & 6.5 & 9.0 \\
\hline BBOA & l & / & I & I & 0.20 & 5.4 & 15.0 \\
\hline OOA & / & / & / & / & 0.74 & 12.7 & 24.1 \\
\hline OPOA & / & / & / & / & 1.9 & 7.7 & 12.2 \\
\hline aqOOA & l & I & I & I & 0.24 & 3.0 & 10.7 \\
\hline \multicolumn{8}{|c|}{ Gaseous pollutants } \\
\hline $\mathrm{CO}\left(\mathrm{mg} \mathrm{m}^{-3}\right)$ & 0.44 & 2.4 & 2.9 & 4.3 & 0.54 & 2.8 & 4.1 \\
\hline $\mathrm{SO}_{2}\left(\mu \mathrm{g} \mathrm{m}^{-3}\right)$ & 10.3 & 48.2 & 55.5 & 55.9 & 6.0 & 23.2 & 21.8 \\
\hline $\mathrm{NO}_{2}\left(\mu \mathrm{g} \mathrm{m}^{-3}\right)$ & 25.3 & 62.7 & 80.0 & 94.5 & 29.0 & 94.1 & 130.8 \\
\hline $\mathrm{O}_{3}\left(\mu \mathrm{g} \mathrm{m}^{-3}\right)$ & 36.1 & 10.7 & 10.2 & 10.5 & 42.6 & 11.0 & 7.6 \\
\hline
\end{tabular}

${ }^{a}$ The aerosol chemical components at the Gucheng rural site were obtained by offline analysis of $\mathrm{PM}_{2.5}$ filter samples and those at the Beijing urban site were obtained by online analysis of NR-PM 1 using a high-resolution aerosol mass spectrometer (HR-AMS).

${ }^{b} \mathrm{OA}$ factors were derived by PMF analysis of HR-AMS data at the Beijing urban site. FFOA-fossil fuel-related OA, COA-cooking OA, BBOA-biomass burning OA, OOA-oxygenated OA, OPOA-oxidized POA, and aqOOAaqueous-phase OOA. 
Table S3. Comparison between the average Mie calculation results acquired by two reported refractive indices of $1.67-0.27 i$ and $1.84-0.21 i$ in previous studies.

\begin{tabular}{|c|c|c|c|c|c|}
\hline Location & Pollution level & $\operatorname{ACS}_{\text {actual }^{a}}{ }^{a}$ & $\mathrm{ACS}_{\text {non-aging }}{ }^{b}$ & $E_{\mathrm{abs}}^{c}$ & Refractive Index \\
\hline \multirow[t]{3}{*}{ Gucheng } & Heavy (Early stage) & 3.09 & 3.01 & 1.02 & \multirow{5}{*}{$\begin{array}{c}1.67-0.27 i \\
\text { (Alexander et al., 2008) }\end{array}$} \\
\hline & Heavy (Middle stage) & 3.97 & 3.53 & 1.12 & \\
\hline & Heavy (Late stage) & 4.43 & 3.18 & 1.39 & \\
\hline \multirow[t]{2}{*}{ Beijing } & Moderate & 2.06 & 1.86 & 1.10 & \\
\hline & Heavy & 3.00 & 2.15 & 1.39 & \\
\hline \multirow[t]{3}{*}{ Gucheng } & Heavy (Early stage) & 3.08 & 3.00 & 1.02 & \multirow{5}{*}{$\begin{array}{c}1.84-0.21 i \\
\text { (Hoffer et al., 2016) }\end{array}$} \\
\hline & Heavy (Middle stage) & 3.97 & 3.51 & 1.13 & \\
\hline & Heavy (Late stage) & 4.47 & 3.13 & 1.43 & \\
\hline \multirow[t]{2}{*}{ Beijing } & Moderate & 2.04 & 1.85 & 1.10 & \\
\hline & Heavy & 2.99 & 2.14 & 1.40 & \\
\hline
\end{tabular}

${ }^{a} \mathrm{ACS}_{\text {actual }}$ represents the absorption cross section of individual POA-containing particles (including core-shell POA-SIA and bare POA) under the actual scenario;

${ }^{b} \mathrm{ACS}_{\text {non-aging }}$ represents the absorption cross section of individual uncoated POA particles (including POA cores without SIA shell and bare POA) under the particle non-aging scenario;

${ }^{c} E_{\text {abs }}$ represents the absorption enhancement factor due to particle aging, i.e., ratio of $\mathrm{ACS}_{\text {actual }}$ to $\mathrm{ACS}_{\text {non-aging. }}$. 
(a) Gucheng rural site

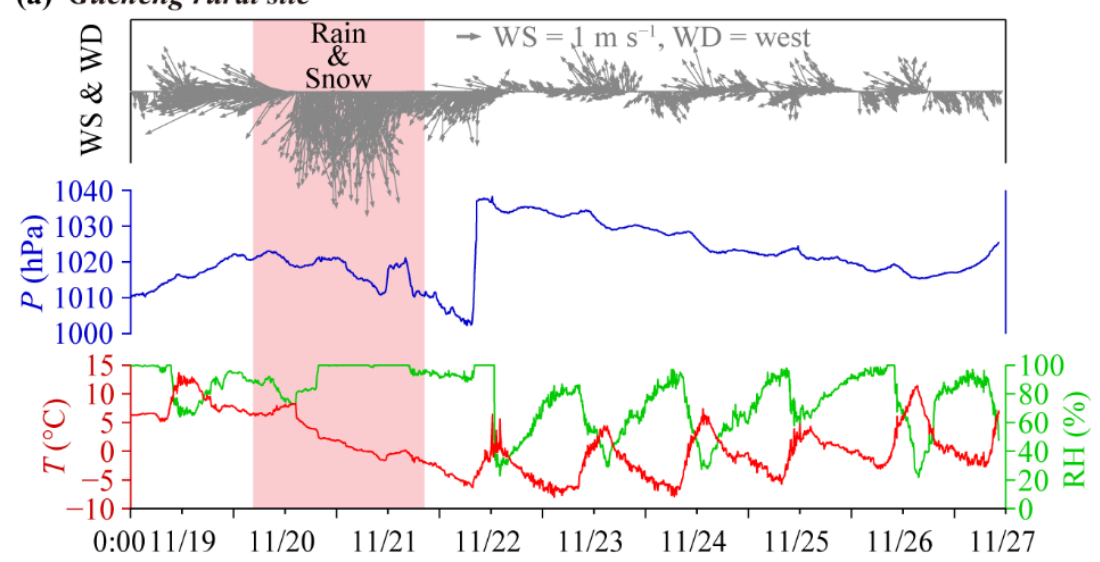

(b) Beijing urban site

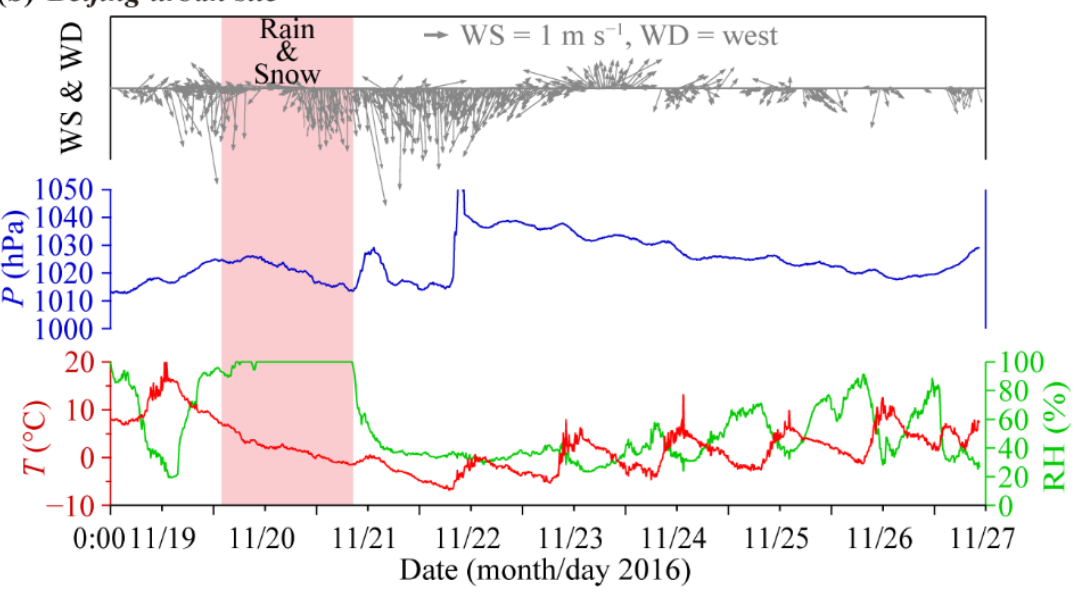

Figure $\mathrm{S} 1$. Time series of meteorological parameters including temperature $(T)$, pressure $(P)$, relative humidity $(\mathrm{RH})$, wind speed (WS), and wind direction (WD) at the (a) Gucheng rural site and (b) Beijing urban site. 
(a) Gucheng rural site

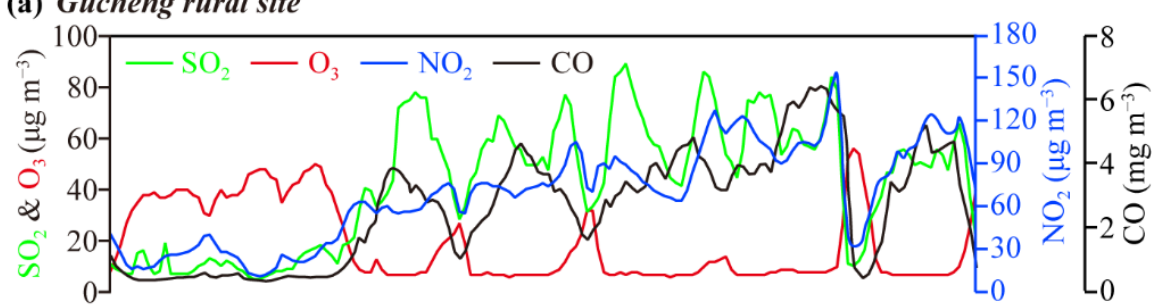

(b) Beijing urban site

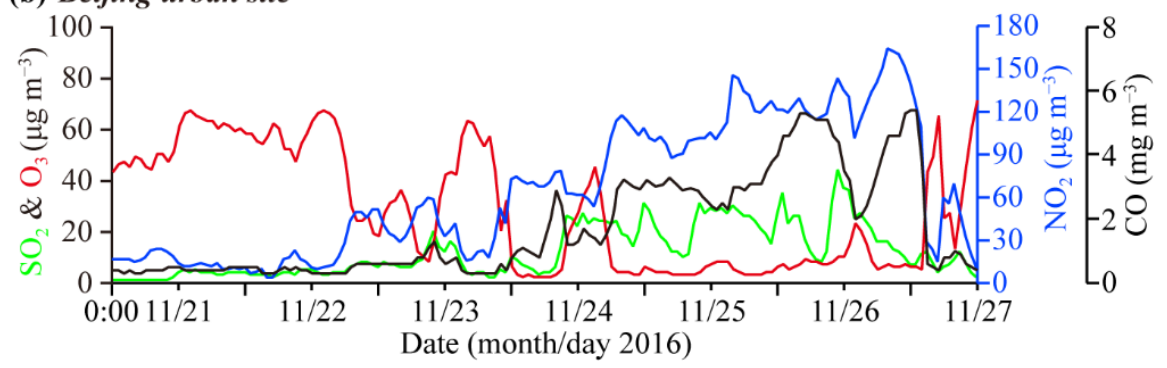

Figure S2. Time series of gaseous pollutants (i.e., $\mathrm{CO}, \mathrm{SO}_{2}, \mathrm{O}_{3}$, and $\mathrm{NO}_{2}$ ) at the (a) Gucheng rural site and (b) Beijing urban site. Data at two monitoring stations (i.e., Dingxing government station: $39^{\circ} 15^{\prime} 42^{\prime \prime}$ N, $115^{\circ} 48^{\prime} 06^{\prime \prime}$ E; Beijing Olympic center station: $40^{\circ} 00^{\prime} 11^{\prime \prime} \mathrm{N}, 116^{\circ} 24^{\prime} 25^{\prime \prime}$ E) close to GC rural and BJ urban sites were downloaded from the website of air quality online monitoring and analysis platform (https://www.aqistudy.cn/). 

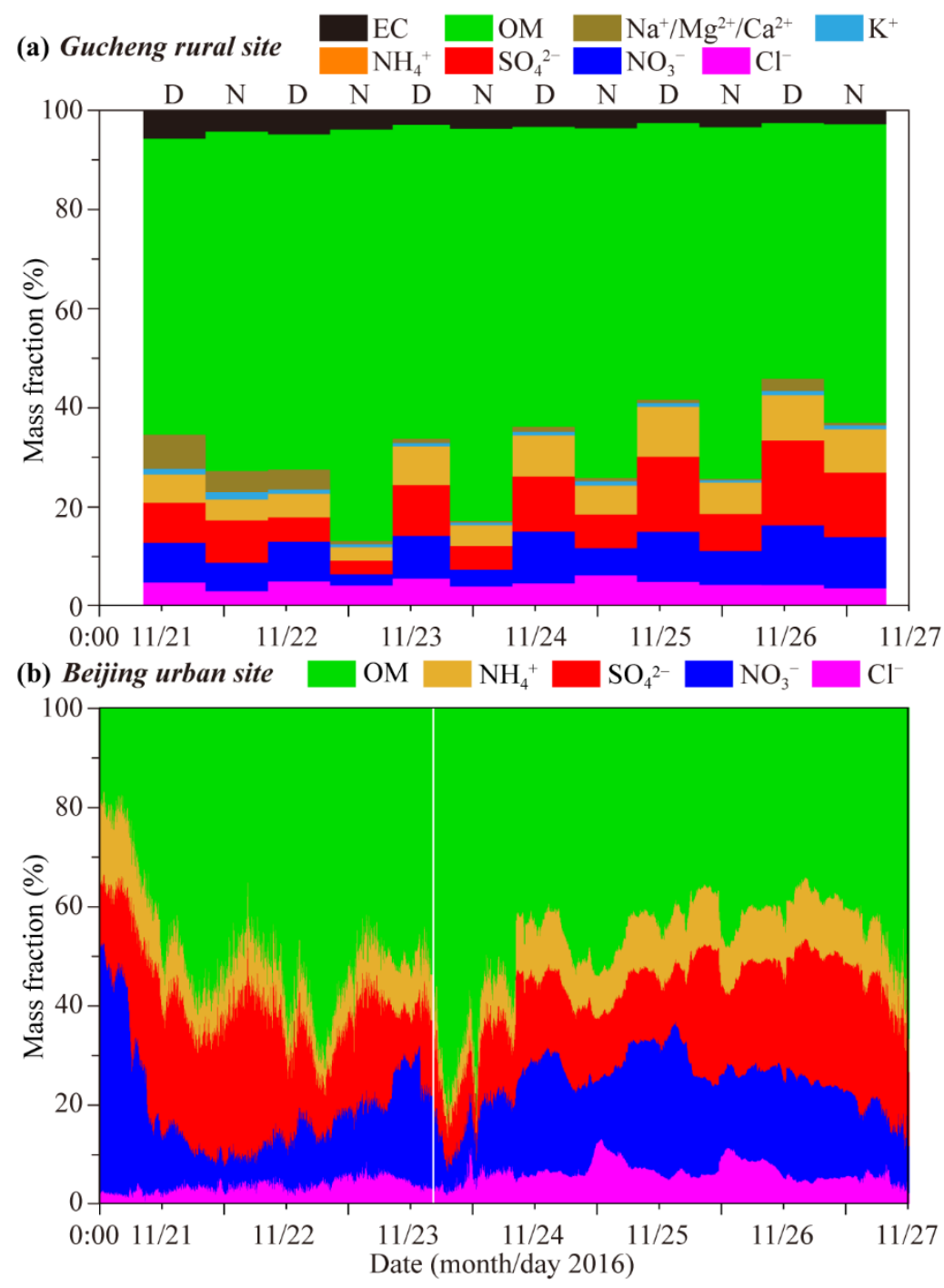

Figure S3. Relative mass fractions of major chemical species in $\mathrm{PM}_{2.5}$ at the Gucheng rural site (a) and in NR-PM at the Beijing urban site (b). 

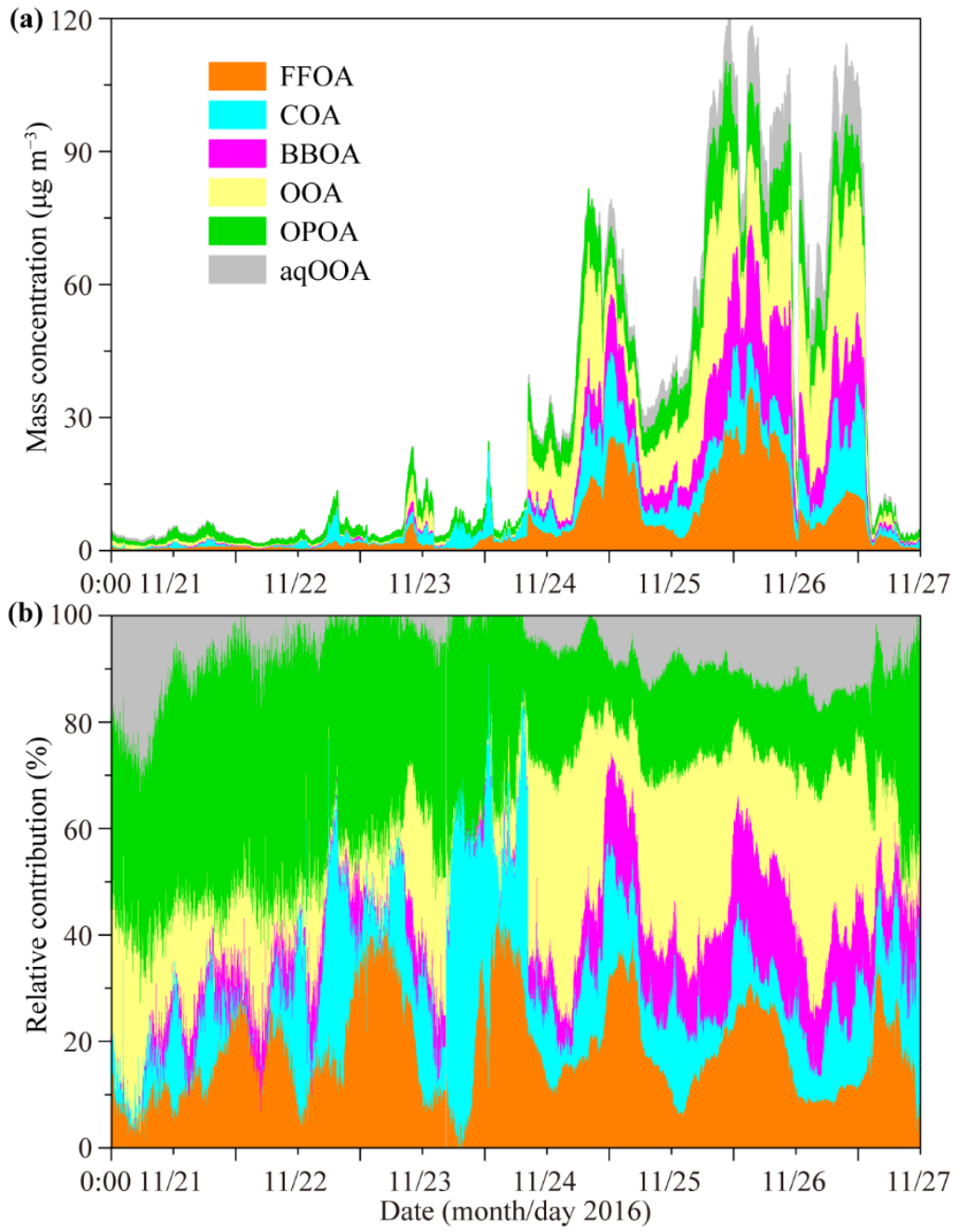

Figure S4. Absolute (a) and relative (b) contributions to organic aerosol (OA) in NR-PM $\mathrm{PM}_{1}$ from different factors (i.e., FFOA-fossil fuel-related OA, COA-cooking OA, BBOA-biomass burning OA, OOA-oxygenated OA, OPOAoxidized POA, and aqOOA-aqueous-phase OOA) at the Beijing urban site. 